BJHS 50(3): 451-472, September 2017. C British Society for the History of Science 2017. This is an Open Access article, distributed under the terms of the Creative Commons Attribution licence (http://creativecommons.org/licenses/by/4.0/), which permits unrestricted re-use, distribution, and reproduction in any medium, provided the original work is properly cited.

doi:10.1017/S0007087417000814

\title{
Regulating cinematic stories about reproduction: pregnancy, childbirth, abortion and movie censorship in the US, 1930-1958
}

\author{
DAVID A. KIRBY*
}

\begin{abstract}
In the mid-twentieth century film studios sent their screenplays to Hollywood's official censorship body, the Production Code Administration (PCA), and to the Catholic Church's Legion of Decency for approval and recommendations for revision. This article examines the negotiations between filmmakers and censorship groups in order to show the stories that censors did, and did not, want told about pregnancy, childbirth and abortion, as well as how studios fought to tell their own stories about human reproduction. I find that censors considered pregnancy to be a state of grace and a holy obligation that was restricted to married women. For censors, human reproduction was not only a private matter, it was also an unpleasant biological process whose entertainment value was questionable. They worried that realistic portrayals of pregnancy and childbirth would scare young women away from pursuing motherhood. In addition, I demonstrate how filmmakers overcame censors' strict prohibitions against abortion by utilizing ambiguity in their storytelling. Ultimately, I argue that censors believed that pregnancy and childbirth should be celebrated but not seen. But if pregnancy and childbirth were required then censors preferred mythic versions of motherhood instead of what they believed to be the sacred but horrific biological reality of human reproduction.
\end{abstract}

In May 1944, Between Two Women was slated to be the next film in MGM's popular Dr Kildare series. The script included a comedic storyline revolving around the characters of Dr Gillespie and Dr Red Adams mistaking twin sisters for the same person as they perform physical examinations on the women. The comedy comes from the fact that one sister is pregnant and the other is not. The scene where Gillespie and his protégé initially discuss the case is illustrative of the way the script mines this scenario and the doctors' competitive natures for comedic purposes:

\footnotetext{
* Centre for the History of Science, Technology and Medicine, University of Manchester, Simon Building, Manchester, UK, M13 9PL. Email: David.Kirby@manchester.ac.uk.

I want to thank organizers Jesse Olszynko-Gryn, Patrick Ellis and Caitjan Gainty; commentator Jessica Borge; and the audience at the 2015 Reproduction on Film conference for helpful comments. Two anonymous reviewers provided invaluable suggestions which have improved the work substantially. A debt of gratitude is particularly owed to Laura Gaither, who read and commented on many versions of this manuscript. This work was supported by the Wellcome Trust (100618) through an Investigator Award entitled Playing God: Exploring the Intersections between Science, Religion and Entertainment Media.
} 
Adams: Dr. Gillespie, I respect you as if you were my own father, I honor your medical knowledge above all men - but Miss Walker is not going to have a baby!

(Gillespie also draws a deep breath, controls himself thoroughly and speaks in a light mood.) Gillespie: To use your own figure of speech, Red - I love you like a son, and you're an exceptionally smart young doctor, but in spite of those facts Miss Walker is going to have a baby! Adams (humors him): No, sir! I applied the Bernhardi-Spinoza test - using frogs!

Gillespie: (exploding) I don't care if you used elephants!

Adams (warming up): The Bernhardi-Spinoza test is infallible.

Gillespie: (in grinning triumph) I know it is! Because I used it on Miss Walker myself!

Adams (right at him): But all four of my frogs proceeded to lay eggs!

Gillespie: (right back at him) All five of my frogs didn't! ${ }^{1}$

The two characters then go to increasingly excessive lengths to prove each other wrong, including the use of greater and greater numbers of frogs for the BernhardiSpinoza pregnancy test (Gillespie uses sixteen frogs at one point). In addition, they use other clinical measurements in order to argue that their diagnosis is correct, including the notion that the patient has 'a blood pressure of 100, perfect blood count, magnificent basal metabolism, and fine glandular balance'. ${ }^{2}$ Near the end of the script they realize their mistake and everyone laughs at how competitive the two doctors had become.

Those familiar with the Dr Gillespie film series, however, will not recognize this storyline. In fact, if you were to watch Between Two Women (1945) you would see that the film does not include anything about twin sisters, the Bernhard-Spinoza test or pregnancy. Why did the filmmakers choose not to include this storyline? Did they consider the storyline too expensive, with its frogs and specialized medical equipment? Did this story clash too much with the other plot elements? Did the narrative perform poorly with test audiences? All of these explanations were valid reasons for why a studio would decide to pull such a story from their film. But the reason the story of the pregnant twin is not in Between Two Women is because Hollywood's self-censorship organization the Motion Picture Producers and Distributors of America (MPPDA), which was popularly known as the 'Hays Office', effectively took the choice of whether to use the narrative out of filmmakers' hands.

The censors at the Hays Office judged that such frank discussions of pregnancy and overt portrayals of pregnancy testing made the whole storyline 'definitely unacceptable material'. ${ }^{3}$ They believed that such candid depictions were inappropriate for both women and men: 'It is our considered judgement that these sequences, discussing

1 Goldbeck, script, 23 May 1944, Production Code Administration archive, Los Angeles (subsequently PCA archive), Between Two Women file, emphasis in original. For the use of frogs in pregnancy testing: Jesse Olszynko-Gryn, 'Frog pregnancy test', in Nick Hopwood, Rebecca Flemming and Lauren Kassell (eds.), Reproduction: From Antiquity to the Present Day, Cambridge: Cambridge University Press, forthcoming in 2018. Bernhardi-Spinoza is a made-up name, but beginning with the famous Aschheim-Zondek test, pregnancy tests were typically named after their inventors. See Olszynko-Gryn, 'The demand for pregnancy testing: the Aschheim-Zondek reaction, diagnostic versatility, and laboratory services in 1930s Britain', Studies in History and Philosophy of Biological and Biomedical Sciences (2014) 47, pp. 233-247.

2 Goldbeck, op. cit. (1).

3 Goldbeck, op. cit. (1). 
pregnancy, the pregnancy tests with various animals, etc., in such detail, are of such a clinical nature as to be entirely unacceptable for mixed audiences in theatres. ${ }^{4}$

This judgement left the studio with only a few choices. They could produce the film using the existing script and release it without the Hays Office's seal of approval. But that course of action was likely a financial kiss of death for a major Hollywood studio because it barred the film from being shown in most movie theatres. ${ }^{5}$ Alternatively, they could negotiate with the Hays Office to come up with a story that would be acceptable to the censors. In this case, the negotiations were futile because there was no way of filming this story without discussing the clinical aspects of pregnancy or pregnancy testing. The only other option was to scrap the entire story and write an entirely new script, which is what the studio ultimately did.

The Catholic Church and other Christian organizations, such as the Woman's Christian Temperance Union (WCTU), the Federation of Churches and the National Council of Churches of Christ in America, played a central role in the creation and administration of these groups. ${ }^{6}$ Hollywood had established the Hays Office in 1922 as a response to protests by these and other religious groups over what they saw as immoral content in movies. ${ }^{7}$ In 1930 studio heads had also agreed to abide by a code of standards called the Motion Picture Production Code that had been written by two prominent Catholics. ${ }^{8}$ MGM was following standard procedure at the time by sending their screenplay to the Hays Office for review and recommendations on how to alter the script so that it met the standards of the Production Code.

In this article I explore the ways filmmakers tried to craft stories about pregnancy, childbirth and abortion and how religiously motivated movie censorship groups modified these narratives before, during and after production in order to tell what they considered to be more acceptable stories about motherhood as a personal, social and cultural act. ${ }^{9}$ Pregnancy, childbirth and abortion were topics of great concern to the

4 Letter from Breen to Mayer, 13 July 1944, PCA archive, Between Two Women file.

5 The potential economic impact of not having a seal of approval from the Hays Office is discussed in Gregory D. Black, Hollywood Censored: Morality Codes, Catholics, and the Movies, Cambridge: Cambridge University Press, 1994; and Leonard J. Leff and Jerold L. Simmons, The Dame in the Kimono: Hollywood Censorship and the Production Code, Lexington: University Press of Kentucky, 2013.

6 For a discussion of the religious underpinnings of movie censorship in the US see Frank Walsh, Sin and Censorship: The Catholic Church and the Motion Picture Industry, New Haven, CT: Yale University Press, 1996; and Lee Grieveson, Policing Cinema: Movies and Censorship in Early Twentieth-Century America, Berkeley: University of California Press, 2004.

7 There are a number of excellent histories that discuss the formation of the Hays Office in 1922; see Black, op. cit. (5); Walsh, op. cit. (6); Leff and Simmons, op. cit. (5); and Ruth Vasey, The World According to Hollywood, 1918-1939, Madison: University of Wisconsin Press, 1997.

8 Martin Quigley was editor of the trade paper Motion Picture Herald. Father Daniel A. Lord was a Jesuit priest. I will use the term 'Production Code' to refer to the Motion Picture Production Code of 1930. For a history of the Production Code see Leff and Simmons, op. cit. (5). A copy of the Production Code can be found in Wilbur R. Miller (ed.), The Social History of Crime and Punishment in America, Thousand Oaks, CA: Sage, 2012, pp. 2225-2227.

9 The topic is far too large to be adequately covered in a single essay. This article will focus exclusively on censors' and studios' negotiations about depictions of reproduction after conception, which includes pregnancy, childbirth and abortion. Censorship discussions related to conception (birth control, in vitro fertilization, etc.) will be dealt with in a separate essay. 
Hays Office, as well as to the other major censorship group in the US, the Catholic Legion of Decency, because of the Christian roots of these organizations. ${ }^{10}$ Human reproduction touches upon many of Christianity's fundamental beliefs, including the meaning of sex, the sanctity of marriage, the sacred status of motherhood, the notion of family, the nature of gender, the purpose of existence and humanity's role on earth. The key for the Hays Office and the Legion of Decency was to make sure the right 'Christian messages' about pregnancy and childbirth were conveyed to movie audiences. Even a storyline as lighthearted as the one in the draft script for Between Two Women was off limits if the censors believed that it would convey an inappropriate message about human reproduction.

The goal of this article is to explore the negotiations between censorship groups and studios in order to understand their struggle over the production of meaning in film texts. Although many scholars refer to the work of the Hays Office and the Legion of Decency as 'censorship', the goal of these groups was generally not to prevent studios from releasing films. Instead, the aim of these groups' activities was to regulate meaning in film content as a means of providing lessons on how to live a moral life or, at the very least, show audiences the dire consequences of leading an immoral life. Subsequent to the development of the Production Code censorship organizations took the approach of closely analysing, commenting upon and recommending changes to the content of every story treatment and script in order to control a film's dialogue, visuals, individual scenes, character motivations, plot points and overall narrative. By altering film scripts before production, censorship organizations could claim to be co-authors of the script, and they would help transmit stories that would have a positive influence on audiences.

Here I am drawing on the work of film scholars, including Lea Jacobs, who argues that the Hays Office did not operate through the restraint of exhibition but instead that censorship was about regulation and 'textual determination'. ${ }^{11}$ Jacobs shows how the Hays Office's mode of censorship 'operated at the level of the text' and 'at the level of representation'. ${ }^{12}$ For the Hays Office and for the Legion of Decency, modifying the text was a means by which they could ultimately control 'the production of meaning' in films. ${ }^{13}$ But the studios, for their part, often resisted censors' attempts to impose moralistic meanings on cinematic texts.

This article is not a historical survey of human reproduction in cinema. There are a number of useful historical surveys that examine this topic. ${ }^{14}$ Rather, this article

10 The organization changed its name to the National Legion of Decency in 1935 but I will refer to as Legion of Decency. For a complete history of the Legion of Decency see Black, op. cit. (5); and Walsh, op. cit. (6).

11 Lea Jacobs, 'Industry self-regulation and the problem of textual determination', Velvet Light Trap (1989) 23, pp. 4-15.

12 Jacobs, op. cit. (11), p. 8.

13 Jacobs, op. cit. (11), p. 4.

14 There are a number of recent books covering the history of depictions of reproduction in cinema and on television; see Heather MacGibbon, Screening Choice: The Abortion Issue in American Film from 1900-2000, Saarbrücken: VDM Verlag, 2009; Kelly Oliver, Knock Me Up, Knock Me Down: Images of Pregnancy in Hollywood Films, New York: Columbia University Press, 2012; Manon Parry, Broadcasting Birth Control: Mass Media and Family Planning, New Brunswick, NJ: Rutgers University Press, 2013; and Parley Ann Boswell, Pregnancy in Literature and Film, Jefferson, NC: McFarland, 2014. 
represents the first comprehensive examination of movie censorship and human reproduction. ${ }^{15}$ Although most histories of reproduction in cinema that cover the period between 1930 and 1960 remark upon movie censorship, few of them explore the issue in depth or examine the religious influences on censor decisions. Scholars most often utilize discussions of censorship to provide an explanation for why there are so few films featuring reproductive issues. This means that research also tends to focus on movies produced after 1960 or films produced before the introduction of the Production Code. Yet MGM's reluctant removal of the pregnancy story from Between Two Women demonstrates why we need to pay as much attention to the cinematic stories about reproduction that are not told as we do to the stories that do make it on to the screen.

The goal of this article is to understand the struggle between filmmakers and censorship groups in the US over the production of meaning in films featuring pregnancy, childbirth and abortion. One method of exploring this battle for control over cinematic stories about human reproduction is to examine the negotiations between the studios and censorship groups over story and script approval. ${ }^{16}$ Therefore this study focuses on the correspondence between studios and censorship groups found in the archives of the Hays Office and the Legion of Decency. Ultimately, this exploration provides new insights about the kinds of story censor groups did, and did not, want told and also about how studios fought to tell their own stories about human reproduction.

I find that movie censors considered pregnancy to be a moral obligation and sacred duty for married women, so they favoured narratives positioning childbirth as a virtuous endeavour. Since procreation was seen as necessary to sanctify the bond between husband and wife they also expressed anxiety that realistic depictions of childbirth would distress young women about the prospect of pregnancy. They also questioned childbirth's entertainment value because they considered childbirth to be an honoured and private female act that would be sullied by allowing men to observe it on the screen. In addition, I demonstrate filmmakers' use of ambiguity to depict stories of abortion on screen in the face of the PCA's strict prohibition against the topic. Ultimately, I argue that censors preferred the absence of pregnancy and childbirth on the screen. But if they were necessary for the story then they preferred mythic versions of motherhood to what they believed to be the sacred but horrific biological reality of pregnancy and childbirth.

\section{Reproduction as a horror film}

In 1930 the Studio Relations Committee (SRC) was the branch of the Hays Office tasked with enforcing the Production Code. The SRC advised studios on how to alter their

15 There are a few scholarly works that pay significant attention to the impact of censorship on depictions of reproduction. See Eric Schaefer, Bold! Daring! Shocking! True! A History of Exploitation Films, 1919-1959, Durham, NC: Duke University Press, 1999; Benjamin Strassfeld, 'A difficult delivery: debating the function of the screen and educational cinema through The Birth of a Baby (1938)', Velvet Light Trap (2013) 72, pp. 4457; and Megan Lynn Minarich, 'Hollywood's reproduction code: regulating contraception and abortion in American cinema, 1915-1952', PhD thesis, Vanderbilt University, Nashville, Tennessee, 2014.

16 This article is part of a larger book project exploring film censorship of science and medicine titled Indecent Science: Religion, Science and Movie Censorship. 
scripts so that they met the standards of the Production Code. The SRC, however, could not force studios to accept their suggestions. This meant that despite their agreement to abide by the Production Code, studios frequently ignored the SRC's recommendations in the early 1930s. ${ }^{17}$ The director of the Hays Office at this time, Colonel Jason Joy, took a particularly lenient approach to the Production Code, which he viewed as a flexible set of guidelines rather than a hard and fast set of rules. Confusingly, the period between 1930, when the Production Code was adopted but laxly enforced, and 1934 is referred to as the 'pre-Code' period.

From the perspective of religious protestors, the Hays Office's failure to rigorously enforce the Production Code meant that movies were just as morally problematic as they had been before its adoption. In response, Will Hays created the Production Code Administration (PCA) as a way to curtail calls by religious groups for a government censorship organization. ${ }^{18}$ This pressure also led the Catholic Church to form its own censorship organization, the Catholic Legion of Decency, in 1933. ${ }^{19}$ The toughminded Catholic Joseph Breen took over as director of the PCA in 1934. Although the Catholic Church was not the only religious voice in these organizations it certainly had an immense influence. ${ }^{20}$ Breen's Catholicism informed his censorship throughout the time that he ran the PCA from 1934 until 1954 and the Legion of Decency explicitly approached movie narratives through the lens of Catholic theology. ${ }^{21}$ Studios' agreement not to release any films without the PCA's seal of approval gave Breen the power he needed to force studios to alter their scripts if they did not meet the standards of the Production Code. ${ }^{22}$ As such, the PCA and the Legion of Decency exerted significant influence over the types of story studios could tell about pregnancy and childbirth.

17 The SRC's ineffectiveness is discussed in Marvin N. Olasky, 'The failure of movie industry public relations, 1921-1934', Journal of Popular Film and Television (1985) 12, pp. 163-170.

18 For a history of the PCA see Black, op. cit. (5). Both the SRC and the PCA were referred to as the Hays Office. However, I will use SRC and PCA to differentiate between the pre-Code Hays Office and the Hays Office after the creation of the PCA in 1934.

19 The Legion of Decency's primary means of censorship was through their film classification system. The Legion's classification system was a guide for Catholic viewers as to what were morally suitable and what were morally questionable films for consumption. They had three levels of classification: A - morally acceptable (with subcategories from I to IV), B - morally objectionable in part and C-condemned. Studios strove for an A classification as they believed that a B or C classification could seriously impact a film's box office if it drove significant numbers of Catholics away from the theatre. Therefore studios often negotiated with this censorship group to avoid a B or C classification. This included sending their scripts or their final films to the Legion for approval or recommendations for cuts. Movie studios took the threat of a Catholic boycott seriously. In the 1930s one in five Americans was Catholic. Catholics were concentrated in the eastern urban areas like Boston and New York that were essential for a successful box office. See Walsh, op. cit. (6), p. 135.

20 See Walsh, op. cit. (6).

21 Breen's Catholic influences are discussed in Walsh, op. cit. (6).

22 Leff and Simmons, op. cit. (5). It should be noted that films approved by the PCA could still face significant censorship trouble from the various regional censor boards including city, state and international censors. 
Catholics considered motherhood to be a sacred state of grace that all women should strive for. ${ }^{23}$ Therefore censors from both the PCA and the Legion of Decency were sensitive to any cinematic portrayals of pregnancy and childbirth that could discourage women from embracing motherhood. Responses to the film Life Begins (1932) were particularly influential on the ways the SRC, and its successor, the PCA, subsequently handled depictions of pregnancy and childbirth. ${ }^{24}$ The SRC told Warner Brothers from the outset that they did not think any film based on the play Birth would be approvable under the Production Code because the story was set entirely within a maternity ward. They warned the studio that even if they could produce an acceptable film it was likely to face severe censorship from local, state and international censor boards. $^{25}$ One SRC censor wanted to prohibit any adaptation of the play because 'women sentimentalize over young motherhood' and he did not want their idealized vision of motherhood sullied by overt discussions about the reality of pregnancy and childbirth. ${ }^{26}$ For this censor, mythic versions of motherhood were acceptable, but he believed that women did not want to see the actuality of motherhood portrayed realistically on motion-picture screens. But another censor convinced SRC director Jason Joy that the play's message about the wonders of motherhood made a film version desirable despite potential issues over direct discussions of pregnancy and childbirth. ${ }^{27}$

Joy told Will Hays that he considered the completed film to be 'experimental' because Life Begins provided the SRC an opportunity to see if regional censors would allow cinematic depictions of pregnancy and childbirth if the regional censors felt that the film was providing important moral guidance for audiences. Unfortunately for Joy, his experiment was a complete failure. A large number of local, state and international censor boards heavily edited the film or banned it completely, as did the British Board of Film Censorship (BBFC) in the UK. ${ }^{28}$ For these censor groups, there was no reason to show the realities of pregnancy and childbirth on movie screens no matter how morally inspiring the film's message might be. The response to this film set the tone for how the SRC (and its 1934 successor the PCA) subsequently approached scripts incorporating pregnancy and childbirth. Their new policy was that pregnancy was not an appropriate subject for cinema, but if it had to be included in a film it was to be

23 Christians in general heeded God's command in Genesis (1:28) to 'Be fruitful and multiply, and fill the earth, and subdue it'. The Papal Encyclical Rerum Novaru in 1895 clearly stated that in the eyes of the Church a woman's primary role was that of wife and mother. The Fourth Catechism of the Catholic Church also asserts that it is a woman's duty to strengthen the family through the production of children.

24 Full details and plot descriptions for the films discussed in this article can be found on the AFI catalogue, at www.afi.com/members/catalog/Search.aspx.

25 Telegram from Joy to Hays, 18 March 1932, PCA archive, Life Begins file.

26 Trotti, review of the play Life Begins, 18 March 1932, PCA archive, Life Begins file.

27 Letter from Joy to Hays, 25 March 1932, PCA archive, Life Begins file.

28 See the Censor Report files, PCA archive, Life Begins file. The BBFC's ban found in Censor Report, England, 17 December 1935, PCA archive, Life Begins file. The BBFC ultimately lifted their ban after the removal of scenes related to childbirth. See James C. Robertson, The Hidden Cinema: British Film Censorship in Action, 1913-1975, London: Routledge, 1993, pp. 57-60. 
mentioned briefly and then relegated to the background. ${ }^{29}$ From the PCA's perspective, the only morally appropriate stories about motherhood were narratives rooted in fantasy where pregnancy did not change women's bodies, childbirth was not painful or dangerous, and every woman desperately wanted a baby. Their restrictive policy did not change until the 1950s.

The PCA's approach of minimizing pregnancy on screen meant that scripts could not include any reminders that pregnancy was a biological process. This meant that studios were prevented from visualizing any external physical changes a woman's body went through during pregnancy. They cautioned the producers of Black Market Babies (1946), for example, that there should be no artificial 'padding' of the women in the film to make them look pregnant. ${ }^{30}$ This restriction was problematic for a visual medium like film. The inability to even show a pregnant woman's abdominal expansion meant that most pregnant women in films between 1930 and the 1950s looked as trim as your average Hollywood actress. ${ }^{31}$ The PCA also policed dialogue about the ways women's bodies changed during pregnancy. The censors found a line 'about putting on weight and eating pickles' in the script for Paid in Full (1950) to be problematic because it implied pregnancy's impact on a woman's body. These concerns about the physical manifestations of pregnancy were not merely because the PCA wanted pregnancy relegated to the background. They also believed that depictions of these physical changes would distress young women and might dissuade them from having babies.

The PCA believed that, even more than pregnancy's accompanying physical changes, the pain of childbirth would deter young women from procreation, so they were vigilant in removing from scripts any indications that childbirth might be a painful experience. One of the major issues that Breen had with the script for Dr. Monica (1934) was its explicit 'talk about the "dangers" of motherhood'. ${ }^{32}$ For example, he told the studio to remove a 'discussion between Dr. Monica and the nurse, in which it is indicated that Mary is "scared to death" at the idea of impending childbirth'. ${ }^{33}$ Breen's explanation as to why dialogue like this was dangerous sums up the PCA's philosophy concerning cinematic presentations of childbirth: 'Audiences have generally come to resent scenes on the screen which present motherhood as a terrifying prospect to the youth of the country'. But even benign phrases like 'she's going to have a baby' were routinely cut by the PCA because they were reminders that having babies was a process and that this process involved pain. For the film Outcast (1937) they suggested that the studio replace the phrase 'she's having a baby' with the phrase 'when her baby is born'. ${ }^{34}$ From their perspective the first phrase was problematic because it connoted the action

29 Breen made the PCA's position on pregnancy clear in a 1937 letter: 'we have made it a practice, where such an indication was absolutely necessary for the plot, to allow the point to be made, and then to ignore it thereafter'. Letter from Breen to Harmon, 31 December 1937, PCA archive, Birth of a Baby file.

30 Letter from Breen to Quigley, 21 August 1945, PCA archive, Black Market Babies file.

31 Letter from Breen to Wallis, 6 October 1948, PCA archive, Paid in Full file.

32 Letter from Breen to Harmon, 31 December 1937, PCA archive, Birth of a Baby file.

33 This and subsequent quote from letter from Breen to Warner, 21 April 1934, PCA archive, Dr. Monica file.

34 Letter from Breen to Cohen, 2 November 1936, PCA archive, Outcast file. 
of childbirth while the second passive phrase was acceptable because it was just a descriptor.

Subtle dialogue was a problem for the PCA, but overt dialogue, explicit sounds and visual depictions of the pain of childbirth were even bigger concerns. The PCA's negotiations over the script for A Child Is Born (1940) are indicative of their approach to minimizing realistic portrayals of childbirth that they believed would distress young women. ${ }^{35}$ The PCA tried to remove any indication from this film that childbirth was a painful process. This included removing specific lines of dialogue indicating the physical difficulties of childbirth, including 'you're the one that'll be shrieking in pain' and 'they get easier all the time'. ${ }^{36}$ But it also included lines of dialogue in which women questioned the necessity of having children, such as, 'Do I have to have them?' In addition, the PCA recommend removing the sounds of childbirth, including the 'offstage screams' of a woman in labour. They even policed stage directions for the actors, such as telling the studio to 'please omit the action of Grace clutching her side, as this will undoubtedly be suggestive of labor pains'.

Despite the PCA's attempts to minimize elements that might make childbirth seem distressing in A Child Is Born, numerous regional censor boards still heavily edited or banned the film because they believed it would frighten women away from pursuing motherhood. British Columbia, for example, rejected the film because ' $\mathrm{t}] \mathrm{he}$ showing of this picture would have the effect of creating a fear in the minds of expectant mothers'. ${ }^{37}$ Part of this response was due to the fact that the studio ignored a number of the PCA's recommendations. Breen made sure to point this out to the studio when he informed them that the BBFC's severe editing of their film in the UK could have been avoided if they had taken his advice. ${ }^{38}$ The BBFC, in fact, had been particularly harsh on the film. For them the film's depictions of childbirth were so disturbing and frightening that they gave it a 'horrific' classification, which meant that a film celebrating the wonders of motherhood could only be seen by adults over eighteen. ${ }^{39}$ From the BBFC's perspective the realities of pregnancy and childbirth were just as disturbing as the monsters in genuine horror films like Frankenstein (1931) and Dracula (1931).

Childbirth was not only painful; it could also be a dangerous activity for both mother and child. Maternal mortality was in decline by the 1930s, the result of rising living standards, antibiotics and blood transfusion services. ${ }^{40}$ But censorship organizations

35 Given the reception of Life Begins it is surprising that the PCA allowed a remake eight years later.

36 All quotes in this paragraph come from letter from Breen to Warner, 9 March 1939, PCA archive, A Child Is Born file.

37 Censor Report, British Columbia, 7 February 1940, PCA archive, A Child Is Born file.

38 Letter from Breen to Bischoff, 2 January 1940, PCA archive, A Child Is Born file.

39 Similarly, the BBFC had previously assigned an A rating to The Mystery of Life (1930) because of its focus on pregnancy and childbirth, which restricted the film to those over sixteen. They had also severely edited the final film to remove any scenes related to childbirth. See Robertson, op. cit. (28), pp. 36-38.

40 On the history of maternal mortality during childbirth see Geoffrey Chamberlain, 'British maternal mortality in the 19th and early 20th centuries', Journal of the Royal Society of Medicine (2006) 99, pp. 559-563; and Salim Al-Gailani, 'Hospital birth', in Nick Hopwood, Rebecca Flemming and Lauren Kassell (eds.), Reproduction: From Antiquity to the Present Day, Cambridge: Cambridge University Press, forthcoming. 
remained leery of film narratives calling attention to the dangers of childbirth. The PCA's response to an expectant mother's utterance of the words 'women die ...' in the script for The Secret of Dr. Kildare (1939) was typical of their approach to this subject. For them this dialogue was unacceptable because 'it overemphasizes the danger of pregnancy'. ${ }^{41}$ Even in the 1950s, foetal mortality remained a constant real-life concern for expectant mothers. ${ }^{42}$ As Breen explained, the PCA took a harsh stand against any depictions of miscarriage: 'With regard to the questions of miscarriage: we simply do not allow such discussions, because, in our judgment - and the studios have unfailingly agreed with us - such discussions are totally unfit for presentation before mixed audiences in theatres.' 43 The PCA ultimately relaxed its stance on miscarriage in the mid-1940s when they were convinced that, if handled carefully, it could provide a legitimate plot point. In the first half of the century, pain-relieving drugs had transformed the experience of childbirth. ${ }^{44}$ These changes led the PCA to soften their general approach to depictions of pregnant women and discussions about childbirth in movies.

\section{Childbirth as entertainment or education?}

One aspect of human reproduction that the PCA and the Legion of Decency were consistently reluctant to allow was explicit visual depiction of childbirth. The Production Code unambiguously prohibited explicit depictions of childbirth: 'Scenes of actual childbirth, in fact or in silhouette, are never to be presented'. ${ }^{45}$ Not only might childbirth scenes frighten potential mothers, but also the PCA considered the act of childbirth to be an intensely private moment not suitable for the immersive medium of cinema. Many moral reformers, such as the Women's Christian Temperance Union, also felt that portrayals of childbirth were disrespectful to the sanctity of motherhood. They believed that allowing men to watch such a private female act jeopardized their respect for women's purity as mothers. ${ }^{46}$ There was also a concern that childbirth scenes were 'indecent' because they might show the woman's genitals. The PCA cautioned studios that they were best off handling childbirth scenes by 'suggestion' rather than through visuals. Any studio ignoring this warning risked having the PCA reject their finished film.

Despite the PCA's restriction, filmmakers still frequently tried to include scenes of childbirth in their entertainment films. In the case of The Birth of a Baby (1938) the entire narrative centred on discussions of childbirth and an actual childbirth was the

41 Letter from Breen to Mayer, 21 August 1939, PCA archive, Secret of Dr. Kildare file.

42 Robert Woods, Death before Birth: Fetal Health and Mortality in Historical Perspective, Oxford: Oxford University Press, 2009.

43 Letter from Breen to Harmon, 31 December 1937, PCA archive, Birth of a Baby file; emphasis in original.

44 See Al-Gailani, op. cit. (40); and Judith Waltzer Leavitt, Brought to Bed: Childbearing in America, 1750 to 1950, Oxford: Oxford University ress, 1986.

45 Miller, op. cit. (8).

46 Alison M. Parker, 'Mothering the movies: women reformers and popular culture', in Francis G. Couvares (ed.), Movie Censorship and American Culture, Amherst: University of Massachusetts Press, 2006, p. 77. 
film's defining scene. ${ }^{47}$ Knowing that they would not be able to garner a PCA seal for their film, the producers appealed directly to the various local, city and state censor boards for approval to show the film in mainstream theatres. The film was banned in New York state with a 1939 court ruling that scenes of childbirth were indecent when presented to patrons of public entertainment'. ${ }^{48}$ But the film was screened in several other places, including Minnesota. The success of these screenings in PCAaffiliated theatres created a major challenge to the PCA's authority. Even though the Production Code forbade portrayals of childbirth, the PCA could not claim that the topic itself was inherently immoral. In addition, too many medical groups had praised this particular film for Breen to risk a public argument with theatre owners over their screenings.

But Breen had to do something otherwise theatres would interpret the PCA's inaction as justification for showing other films that went against the Production Code but were not necessarily immoral. The PCA solved this issue by constructing an official division between films that were appropriate for 'entertainment' and those that were 'educational'. ${ }^{49}$ Film scholar Benjamin Strassfeld shows how this division was a clever means by which the PCA could maintain its control over exhibition. ${ }^{50}$ The distinction between entertainment and education led to the physical segregation of the places where these two types of film could be shown. This separation of exhibition spaces meant that rather than having to judge the morality of a film itself, the PCA could now judge the morality of the film's exhibition context. Films that the PCA deemed 'educational' could not be screened in mainstream theatres and instead could only be shown in churches, schools and lecture halls. In one exhibition context childbirth was exploitative and immoral, but in another theatre space the topic was uplifting and moral.

The PCA argued that a film's story was never harmed by the removal of childbirth scenes. If a plot required the birth of a child then they felt it was adequate to just mention that a baby had been born. The case of Forgotten Village (1941) demonstrates how the censors came to this conclusion because they embraced a simplistic conception of motion pictures as merely linear stories with easily understandable narratives. The film focuses on a rural Mexican village where the government hopes that modern medicine will replace a native herb doctor. The PCA judged the finished film to be satisfactory except for a lengthy childbirth scene that they requested be removed before approval. According to Breen this should have been an easy decision for the studio because removing these scenes did not hurt the film's story in any way: 'It is our considered unanimous judgment, here, that the eliminations made to bring this picture within the provisions of

47 The material in this and the subsequent paragraph concerning The Birth of a Baby comes from Strassfeld, op. cit. (15).

48 Laura Wittern-Keller, Freedom of the Screen: Legal Challenges to State Film Censorship, 1915-1981, Lexington: University Press of Kentucky, 2008, p. 181.

49 The PCA's distinction between entertainment and education is also discussed in Martin S. Pernick, The Black Stork: Eugenics and the Death of 'Defective' Babies in American Medicine and Motion Pictures since 1915, Oxford: Oxford University Press, 1996; and Kirsten Ostherr, Medical Visions: Producing the Patient through Film, Television, and Imaging Technologies, Oxford: Oxford University Press, 2013.

50 Strassfeld, op. cit. (15). 
the Production Code do not, in any way, seriously detract from the general importance and value of this film'. ${ }^{51}$ The studio, however, disagreed with Breen's artistic assessment concerning the significance of this scene for the film. So they decided to release the film without the PCA's approval, knowing that the film might run into censorship issues with regional censor boards; which it did.

The New York censor board, for example, banned the film because of the inclusion of this childbirth scene. The film's distributor protested to the State Board of Regents about the censor board's verdict. The Board of Regents lifted the ban and allowed the uncut film to be shown in New York. Famed New York Times film critic Bosley Crowther took the Board of Regents' decision as a 'cause for rejoicing'. ${ }^{52}$ Unlike Breen and the PCA, who considered the scene to be inessential, Crowther believed that 'a sustention of the censors' prudish orders would have desecrated a work of pictorial art'. Crowther considered the childbirth scene essential because it contributed to the film's thematic exploration of 'the human cycle of birth, daily toil and the inexorable mystery of death'. But for the censors the film was merely the story of a teacher helping to bring modern medicine to a rural village. From their perspective it was an unpleasant scene that was entirely unnecessary for audiences to understand this simple plot.

The PCA may have excluded explicit depictions of childbirth from mainstream films, but from the studios' standpoint the notion of what constituted an 'actual' depiction of childbirth was still contentious in the 1950s. In the case of Paid in Full (1950), for example, the PCA had worked extensively with the studio to produce an acceptable shooting script from what they saw as morally difficult material about an adulterous affair. Yet when the studio presented the final film for approval it was rejected immediately not because the studio had handled the controversial adultery material inappropriately but rather because of 'scenes in the maternity hospital which show the doctor lifting forth the new-born baby, and holding it in the air'. ${ }^{53}$

The film's producer, Hal Wallis, argued that the scene was acceptable because 'the audience does not see the actual outward movement of the child from its mother's womb'. ${ }^{54}$ Breen pointed to the realism of the scene as his reason for considering it to be an overt depiction of childbirth: 'These scenes are so graphically presented that you almost feel, as you watch them, that you are actually within a few feet of a woman giving birth to a child'. For Breen, cinema was such an immersive medium that a scene shot with such 'stark realism' made audiences believe they were witnessing an authentic birth whether they saw the baby leave the womb or not. In the end the PCA allowed the scene to be kept if the studio edited it to remove shots of the doctor lifting the newborn in the air; a now standard, almost cliché, trope in depicting childbirth.

51 Letter from Breen to Wobber, 21 March 1941, PCA archive, Forgotten Village file.

52 This and all subsequent quotes in this paragraph from Bosley Crowther, "“The Forgotten Village," a wellphotographed film depicting Mexican life, at the Belmont Theatre after censor trouble', New York Times, 19 November 1941, p. A27.

53 Letter from Breen to Hallis, 14 December 1948, PCA archive, Paid in Full file.

54 All quotes in this paragraph come from letter from Breen to Johnston, 10 December 1948, PCA archive, Paid in Full file. 
Despite the PCA's segregation of films featuring childbirth as 'educational', some filmmakers still tried to get films featuring childbirth into mainstream theatres by calling them 'semi-documentaries'. The producers of Forgotten Village originally tried to get PCA approval for their childbirth scene by arguing that the film, despite being fictional, was educational and 'documentary in style'. ${ }^{55}$ The distributors of the French film The Case of Dr. Laurent (1957) were equally unsuccessful when they tried using this same rationale. The film concerned a supposedly scientifically proven method of 'natural' childbirth that did not require anesthesia (Figure 1). ${ }^{56}$ The PCA rejected The Case of Dr. Laurent because of its 'detailed, prolonged, and full-length view of an actual childbirth', as well as its overly clinical discussions of reproduction because they violate the Production Code's prohibition of 'sex hygiene'. 57 They made this decision despite the fact that, according to the PCA's new director, Geoffrey Shurlock, the staff found the film inspiring:

[The filmmakers] have managed to handle the subject so honestly that, believe it or not, none of us were shocked by the scene of the childbirth. This goes for the full staff and the two girls who were taking notes - one of whom is on the way to becoming a mother herself in a few months. This girl particularly thought the film was just wonderful. ${ }^{58}$

Ultimately, the PCA refused the film a certificate as a matter of policy. The Production Code forbade depictions of childbirth. There were no exceptions, no matter how sensitively the film treated the topic.

The PCA's decision, however, contrasted with the judgement of the Legion of Decency, who commended the film for its 'educational value'. ${ }^{99}$ The Catholic Church approved of 'natural childbirth' as a means of removing pain from the birthing process. ${ }^{60}$ The film's message also appealed to the Church because it showed 'that childbirth need not be frightful or frightening'. ${ }^{61}$ The film had also received highly favorable reviews in prominent newspapers. These positive responses caused the current PCA director, Geoffrey Shurlock, to reassess the PCA's decision, 'The more I think over the Dr. Laurent situation, and consider the favorable reviews the picture has received, with specific commendation of the treatment of the birth scene, the more I am convinced that we goofed in rejecting it'. ${ }^{62}$ Not only did he think they should reconsider this film, but he also thought it was time that the PCA re-evaluated their entire policy concerning pregnancy and childbirth.

55 Letter from Kline to Breen, undated, PCA archive, Forgotten Village files.

56 See Salim Al-Gailani, this issue.

57 Letter from Shurlock to Ginsberg, 19 June 1958, PCA archive, The Case of Dr. Laurent file.

58 Letter from Shurlock to Clark, 20 June 1958, PCA archive, The Case of Dr. Laurent file.

59 Letter from Little to Cowles, 24 July 1958, Legion of Decency archive, Washington, DC, The Case of Dr. Laurent file.

60 In 1956 Pope Pius XII gave a speech to gynaecologists in Rome praising natural childbirth. Pope Pius XII, 'Painless childbirth', 8 January 1956, Legion of Decency archive, Washington, DC, The Case of Dr. Laurent file.

61 'Birth without tears', Commonweal, 4 July 1958, p. 376.

62 Shurlock had replaced Breen as head of the PCA in 1954. Quote found in letter from Shurlock to Clark, 3 July 1958, PCA archive, The Case of Dr. Laurent file. 

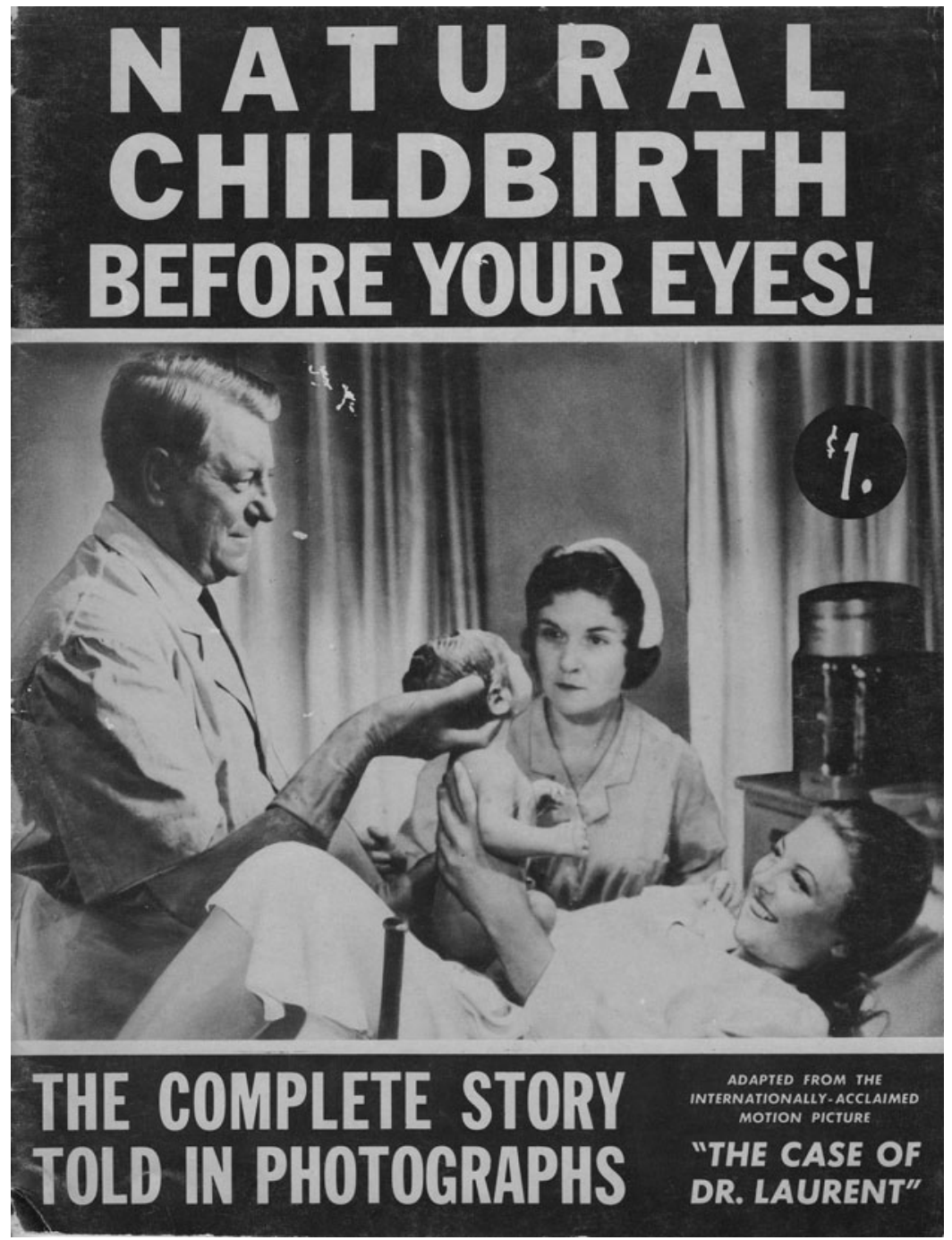

Figure 1. Front cover of a promotional photobook accompanying the film The Case of Dr. Laurent (1957). (C) 1958 by Ben Alder Advertising Service, Inc.

For one thing, Shurlock and his censors could not come to an agreement as to what the term 'sex hygiene' actually referred to. This was a problem given that they had used the term over the previous thirty-eight years as the means by which to determine the appropriateness of a wide variety of subjects, including pregnancy. ${ }^{63}$ In addition, Shurlock pointed out that the Production Code only required that they be handled 'with discretion

63 Letter from Shurlock to Clark, 20 June 1958, PCA archive, The Case of Dr. Laurent file. 
and restraint and within the careful limits of good taste'. ${ }^{64}$ Previously, the PCA interpreted this wording to mean that movies should never show childbirth. However, this particular film's overwhelmingly positive reception forced him to wonder if their previous interpretation was wrong. He now believed that they should allow scenes of childbirth as long as they were done in good taste. In the end Shurlock decided to reverse his decision on The Case of Dr. Laurent and grant the film a PCA seal of approval. ${ }^{65}$ Shurlock's reassessment of the PCA's policy on pregnancy and childbirth and the Legion of Decency's approval of the film ultimately resulted in a less restrictive approach to these topics by both organizations with films produced after 1958 .

\section{Ambiguity and abortion}

Regulating depictions of pregnancy and childbirth was important to the PCA and the Legion of Decency because they wanted to preserve perceptions of motherhood as a celebratory act.

Abortion, on the other hand, was an overt rejection of motherhood. Therefore it was the one aspect of post-conception human reproduction that these censorship organizations prevented from reaching screens in any form.

Abortion was not included as part of the original Production Code in 1930 and did not appear explicitly in the document until 1951; until then the subject was not technically prohibited for inclusion in motion pictures. ${ }^{66}$ They did not include abortion in the Production Code because they believed that studios tacitly understood that this topic was forbidden. ${ }^{67}$ As Breen pointed out to one studio who argued that their story should be approved because abortion was not mentioned in the Production Code, 'We have taken the stand that this one subject, which while not specifically mentioned in the Code, is not suited for screen dramatization, before mixed audiences, in theatres' ${ }^{68}$ This long-standing ban on the subject did not, however, prevent studios from attempting to incorporate abortion narratives into their scripts.

The PCA's severe stance meant that from 1934 until the early 1950s abortion was not mentioned in films. But in the pre-Code era between 1930 and 1934 studios were often able to perform narrative gymnastics in order to tell some semblance of the story they wanted to tell about abortion. The Production Code's conventions allowed for what film scholar Ruth Vasey calls the 'principle of deniability'. ${ }^{69}$ As her husband, film historian Richard Maltby, explains, the Production Code 'permitted producers to deny responsibility for a movie's content, through a particular kind of ambiguity, a textual indeterminacy that shifted the responsibility for determining what the movie's content

64 Letter from Shurlock to Clark, 1 July 1958, PCA archive, The Case of Dr. Laurent file.

65 Letter from Shurlock to Brandt, 9 July 1958, PCA archive, The Case of Dr. Laurent file.

66 Modifications to the Production Code can be found at productioncode.dhwritings.com/ multipleframes_productioncode.php.

67 Leff and Simmons, op. cit. (5), p. 173.

68 Letter from Breen to Johnston, 15 April 1949, PCA archive, The Doctor and the Girl file.

69 Vasey, op. cit. (7), p. 107. 
was away from the producers to the individual spectator'. ${ }^{70}$ There were a substantial number of films incorporating abortion storylines before the formation of the PCA in 1934. But abortion-based films of the pre-Code period were not straightforward narratives about the topic. These films relied on veiled language and ambiguous scenes. This ambiguity prevented the censors from removing these storylines because they could not prove that they were explicitly about abortion. But long-standing narrative codes and conventions made it clear to mature audiences what the stories were about. ${ }^{71}$ For the SRC it became a Sisyphean battle trying to control the ambiguity in these stories so that they could not easily be read as narratives about abortion.

The negotiations between Warner Brothers and the SRC about the script for 1931's Alias the Doctor demonstrate how the censors' attempts to control the ambiguity in these stories were doomed to fail. The original script involved a playboy medical student named Stephan performing an unspecified operation on his girlfriend before he had his medical degree. The girl dies from the botched operation and his foster brother decides to protect him by taking the blame for the operation. The SRC found the initial script problematic because, from their perspective, 'there would be no possibility of misunderstanding the nature of the operation proposed in Alias the Doctor' as anything other than an illegal abortion. ${ }^{72}$ Because the details of the operation were unclear, producer Daryl Zanuck claimed that he was 'amazed and bewildered' that the censors considered the operation to be an abortion. ${ }^{73}$ Zanuck disingenuously stated that the only way the SRC could find an abortion in the text was if they 'read it into the script' themselves. The SRC was reading an abortion into the script, but their reading was based on their knowledge of how studios frequently used veiled language to bypass censorship restrictions. From their perspective there was 'no other possible interpretation' despite the ambiguity of the dialogue. ${ }^{74}$

To remove any ambiguity from the situation Zanuck agreed to provide a specific cause for the operation. In the revised script the two lovers argue and Stephan pushes his girlfriend down the stairs. For Zanuck this removed any possible way to read an abortion into the scene:

I think that if you read this, you will agree with me that certainly there cannot be any complaint when I definitely state the cause of the accident and the cause of the operation, and stress the fact that the operation was not illegal but that he, as a medical student, had no right to even set a broken finger before graduation. ${ }^{75}$

The studio's inclusion of a specific non-abortion operation led the SRC to believe that it had won its battle with narrative ambiguity. The censors were satisfied that 'the dangerous element has been taken care of' and that the film's operation 'isn't an abortion now

70 Richard Maltby, 'More sinned against than sinning: the fabrications of "pre-code cinema", Senses of Cinema (2003) 29, at sensesofcinema.com/2003/feature-articles/pre_code_cinema.

71 See MacGibbon, op. cit. (14).

72 Letter from Joy to Zanuck, 23 November 1931, PCA archive, Alias the Doctor file.

73 This and subsequent quote from letter from Zanuck to Joy, 24 November 1931, PCA archive, Alias the Doctor file.

74 Letter from Joy to Zanuck, 25 November 1931, PCA archive, Alias the Doctor file.

75 Letter from Zanuck to Joy, 25 November 1931, PCA archive, Alias the Doctor file. 
and couldn't possibly be considered such'. ${ }^{76}$ But no matter how much the SRC tried to remove ambiguity from this film, contemporary audiences' familiarity with these narratives would lead them to see the operation as a failed abortion. Well-worn narrative codes implied that Stephan's explanation about pushing the girlfriend down the stairs was merely his attempt to conceal an illegal abortion. ${ }^{77}$

The SRC's weak attempt to eliminate ambiguity from cinematic abortion narratives played a role in the organization's dissolution in 1934 and its replacement by the PCA. Men in White (1934) was based on a controversial play whose plot relied heavily on abortion. ${ }^{78}$ Although there were no overt references to abortion in the original script, the SRC found the story to be in violation of the Production Code because it contained the 'definite suggestion that Barbara's illness is brought about by an attempt at abortion'. ${ }^{79}$ As with Alias the Doctor, the SRC suggested that the studio overcome this 'definite suggestion' by including a specific non-abortion reason for Barbara's surgery, like a failed suicide attempt. The studio rejected the SRC's suggestions to specify the character's illness because ambiguity worked in their favour. They believed that most audiences would interpret the story as being about abortion, and the abortion storyline is what had made the play incredibly popular. Ultimately, the SRC gave their tentative approval to a negotiated final script but they insisted on seeing the filmed version before they would clear the picture. ${ }^{80}$

The SRC identified several lines of dialogue in the filmed version that they felt strongly insinuated an abortion. Although abortion is not mentioned in a doctor's line - 'Some of our laws are hard to understand at times they work cruel hardships but they are laws, and reputable doctors and hospitals obey them' - it would have been clear to audiences in 1934 which laws the doctor was referring to. Hence the SRC had the studio cut the line from all existing film prints. ${ }^{81}$ The studio balked, however, at the SRC's insistence that they remove the word 'peritonitis' from the film. The studio argued that that the word was just a generic medical term for an inflammation of abdominal tissue resulting from any number of causes. For the SRC, however, that term unquestionably signified a botched abortion and so it had to be removed. ${ }^{82}$

According to Will Hays the SRC's goal was to eliminate any 'definite suggestion of abortion' from the film. To that end he felt that most audiences would not read the edited film's ambiguous dialogue as a story about abortion:

76 Letter from Joy to Hays, 20 January 1932, PCA archive, Alias the Doctor file.

77 Ireland's film censors removed this scene because they insisted that the operation was an abortion. See Kevin Rockett, 'Protecting the family and the nation: the official censorship of American cinema in Ireland, 1923-1954', Historical Journal of Film, Radio and Television (2000) 20, pp. 283-300, 289.

78 For discussion of the play see Estelle Manette Raben, 'Men in White and Yellow Jack as mirrors of the medical profession', Literature and Medicine (1993) 12, pp. 19-41. See also Susan E. Lederer, 'Repellent subjects: Hollywood censorship and surgical images in the 1930s', Literature and Medicine (1998) 17, pp. 91-113; and Minarich, op. cit. (15).

79 Letter from Breen to Mannix, 23 December 1933, PCA archive, Men in White file.

80 Letter from Breen to Mannix, 8 January 1934, PCA archive, Men in White file.

81 Memo from Kelly to Film Department, 2 April 1934, PCA archive, Men in White file.

82 Memo from Hays to Mackenzie, 4 April 1934, PCA archive, Men in White file. 
The cause of the girl's illness in the picture will have to be guessed at; the lines seem to suggest any one of a dozen things ... It is believed $95 \%$ of the audiences who see the picture will have to guess at what causes the tragedy. Some will think she tried to commit suicide, the others - none can tell. ${ }^{83}$

Nonetheless, 'a storm of protest broke out' at the time of the picture's release amongst moral reformers and religious groups. Men in White was one of the first films condemned by the newly formed Legion of Decency. ${ }^{84}$ It was also central in religious protestors' arguments about the need for a more effective Hollywood self-censorship organization, which eventually led to the formation of the PCA. Years of veiled abortion films had primed audiences in 1934 to see any story of an illicit love affair followed by a dangerous illness as indicative of an abortion.

For all the unmistakably coded situations, imagery and dialogue that still remained in the heavily censored film, the SRC would have been just as successful in controlling the film's message had they just allowed the studio to tell a straightforward abortion narrative. Film scholar Heather MacGibbon argues, in fact, that the SRC's approach of glossing over the specifics surrounding abortion was more harmful because it traumatized viewers by mystifying the unknown: 'Without representation the audience imagines the worst possible evil, just as in many horror films where the monster is not seen directly because the imagination created a monster even more frightening than special effects could at the time. ${ }^{\prime 85}$ As useful as open stories might be in demystifying abortion, the vitriolic response to Men in White demonstrated how strongly opposed religious groups were to any inclusion of abortion in motion pictures. The SRC's lenient policy of massaging scripts to remove 'definite suggestions' of abortion became the PCA's strict policy 'not to allow any suggestion of abortion or even any discussion with regard to it' ${ }^{86}$ Using ambiguity to tell a story about abortion was no longer possible under the PCA.

One studio was able to include an overt representation of an abortion during this period because the PCA convinced itself that a self-induced miscarriage was not an abortion. The story of Leave Her to Heaven (1945) included the pregnancy of a possessive woman named Ellen. Ellen comes to despise her unborn baby and she decides to induce a miscarriage by intentionally falling down the stairs. Her original motivation for inducing the miscarriage was because she was concerned that the unborn child was deformed. ${ }^{87}$ The PCA told the studio to change this motivation in order to 'avoid any flavor that is normally connected with what could be termed "abortion". 88 The studio then changed Ellen's motivation to a concern that the pregnancy had made her unattractive to her husband. But as with the previous script the PCA felt that this

83 Letter from Hays to Rubin, 4 January 1934, PCA archive, Men in White file.

84 Letter from Breen to Harmon, 31 December 1937, PCA archive, Birth of a Baby file.

85 MacGibbon, op. cit. (14), pp. 179-180.

86 The PCA made it clear that 'the Policy of the PCA has been at all times not to allow any suggestion of abortion or even any discussion, with regard to it'. Letter from Breen to Harmon, 31 December 1937, PCA archive, Birth of a Baby file.

87 On mothers' concerns about giving birth to 'defective' babies see, for example, Pernick, op. cit. (49); Leslie J. Reagan, Dangerous Pregnancies: Mothers, Disability, and Abortion in Modern America, Berkeley: University of California Press, 2010; Patrick Ellis, this issue.

88 Letter from Breen to Joy, 12 December 1944, PCA archive, Leave Her to Heaven file. 
particular motivation gave the self-induced miscarriage the 'flavor of abortion'. ${ }^{89}$ Breen suggested that this difficulty could be avoided if they 'established that her reason for murdering the child is that she thinks that the newborn will replace her husband's affections [for her]'.90

In this case the PCA are making a fascinating distinction between what constitutes an abortion and what constitutes a murder. They seem to be implying that it is an abortion if the woman's motivation for inducing a miscarriage is based upon the mere fact of being pregnant (for instance, the possibility of a deformed child or the physical changes associated with pregnancy). But if her motivation involved a particular individual that she felt had wronged her, in this case the unborn child whom she believed was stealing her husband's love, then the PCA considered it murder because she is killing a specific 'person'. Regardless of their reasoning, their subtle distinction reveals the lengths they went to to remove any suggestion of abortion from cinematic narratives. After the Men in White uproar they would not allow any narrative uncertainty that audiences might interpret as an abortion. But if the PCA convinced itself that this was unambiguously a murder, not an abortion, then they could deal with the story the same way they treated any murder. By reclassifying her actions as murder, then, the story became acceptable because the murderer received a suitable punishment for her crime. ${ }^{91}$

By the late 1940s, however, the PCA's blanket prohibition of stories even hinting at abortion became difficult to sustain. Moral standards had loosened during the war. The influx of returning soldiers led to a rise in the number of births out of wedlock that removed some of the stigma from abortion. ${ }^{92}$ The topic began to appear in popular magazines and public discussion. ${ }^{93}$ Studios wanted to take advantage of this heightened public awareness. Several times studios argued that the PCA should allow them to produce a film because their narrative portrayed abortion in a negative light. In the case of the unproduced 1950 script Unborn the scriptwriter argued that because the film was an 'exposition of the abortion racket' he believed it would garner vocal backing 'from public groups interested in this great social cancer'. ${ }^{94}$ Breen told him that while it might seem logical that anti-abortion groups would support cinematic stories showcasing the evils of abortion, the reality was that the writer would instead run into 'a great deal of opposition from the various groups he thinks should be in support of it'.

The producer of The Doctor and the Girl (1949) made a similar argument to the PCA, saying that the group should approve his script because 'the entire theme is calculated to serve as a deterrent'. ${ }^{95}$ While Breen agreed that the story possessed 'all the moral values

89 Letter from Breen to Joy, 2 March 1945, PCA archive, Leave Her to Heaven file.

90 Letter from Breen to Joy, 12 December 1944, PCA archive, Leave Her to Heaven file.

91 The Legion of Decency also did not consider her actions to be an abortion. They classified the film A-II.

92 See Leslie J. Reagan, When Abortion Was a Crime: Women, Medicine, and Law in the United States, 1867-1973, Berkeley: University of California Press, 1997.

93 Leff and Simmons, op. cit. (5), p. 173.

94 This and subsequent quote from Breen, memo for the files, 16 June 1950, PCA archive, Unborn file, original underlining.

95 This and subsequent two quotes from letter from Breen to Johnston, 15 April 1949, PCA archive, The Doctor and the Girl file. 
in the world', he was steadfast in his decision not to approve this film because 'for us to approve it in this particular picture would establish a precedent for the treatment of abortion in other films'. It was a catch-22 situation for Breen. He believed that there were morally acceptable stories about abortion, but if the PCA allowed these stories to be produced they would legitimate the topic. They would then 'have the subject of abortion injected into fifty pictures presented for our approval'. From Breen's perspective the only appropriate cinematic story about abortion was no story.

Unfortunately for Breen, the MPAA's president, Eric Johnston, was an Episcopalian who didn't share the same views on abortion in film as the Catholic Breen. ${ }^{96}$ The studio appealed the PCA's decision directly to Johnston. They argued that abortion was not explicitly forbidden in the Production Code and that the topic could be rendered acceptable if the script handled it with 'good taste'. Johnston agreed that the film's message made it an appropriate story for the screen so he insisted that Breen work out a solution with the studio. The Doctor and the Girl signalled the return of veiled dialogue in abortion narratives as Breen compromised by allowing them to refer to 'an illegal operation'. ${ }^{97}$ Breen's prediction that the PCA would be inundated with stories about abortion if they approved this film turned out to be correct. Despite the vagueness of the phrase 'illegal operation', studios realized that the PCA had just allowed an abortion narrative on the screen.

The next script involving abortion to come across the PCA's desk was for the film Beyond the Forest (1949). The PCA decided that since The Doctor and the Girl received so little negative feedback they would employ the same strategy for concealing the film's abortion plot using veiled language, such as placing a sign on the abortionist's door referring to his services as a 'Psychological Consultation'. ${ }^{98}$ In this case, however, ambiguity did not prevent a flood of hostile responses to the film, including condemnation by the Legion of Decency. The Legion's C classification had a major impact on the film's box office, which forced the studio to negotiate cuts to the film in order to be reclassified as a B. The Legion rejected any narrative uncertainty as far as abortion was concerned. One of the changes they forced on the studio was the replacement of the ambiguous 'Psychological Consultation' sign for an unambiguous 'Lawyer's Office' sign. ${ }^{99}$

Breen felt burned by his experience with Beyond the Forest. He resented the Legion's criticism of his judgement that the film had appropriately obscured its abortion scenario. But he was more sympathetic to the Legion's position on abortion narratives than he was to Johnston's desire for compromise with the studios. When the script for Detective Story (1951) arrived on his desk he felt justified in taking a hard-line stance; there was to be no compromise this time. He insisted that Paramount remove all references to abortion and change the illegal activity in the script from abortion to an illegal 'baby farm'. However, Breen could no longer argue that the PCA never allowed abortion narratives. The film's director, William Wyler, wondered why the PCA approved abortion stories in

96 Johnston had replaced Will Hays as president of the MPDA in 1945. One of his first acts was to change the name from the MPDA to the Motion Picture Association of America (MPAA).

97 Leff and Simmons, op. cit. (5), pp. 173-174.

98 Seal of approval, 23 August 1949, PCA archive, Beyond the Forest file.

99 Walsh, op. cit. (6), pp. 242-244. 
two recent films but they would not allow him to produce a film that clearly demonstrated the evils of abortion. ${ }^{100}$ Wyler went public with his complaints about the PCA. In an interview with the New York Times he called the Production Code old-fashioned and asked, 'Why not discuss reality?'101 But 'reality' was what the censors feared most in cinematic stories. Groups like the PCA and the Legion of Decency still preferred idealized mythic worlds where abortion did not need to exist. They wanted their movies to tell unambiguous stories with easily understood moral messages. That wasn't reality and societal changes slowly chipped away at the PCA's authority throughout the 1950s.

\section{Conclusions}

The Case of Dr. Laurent was a significant film for both the PCA and the Legion of Decency because their deliberations led them to change their policies towards the depiction of pregnancy and childbirth in 1958. But both groups had been under pressure to change their approaches to reproductive issues in movies since the late 1940s. Before the Second World War the Production Code's provisions matched the values of the American public and American churches. But after the war there was a general loosening of moral standards and the American public began to question these groups' control over movie content. ${ }^{102}$ Protestants also began to complain that the Code was biased against non-Catholic themes. ${ }^{103}$ In addition, the Supreme Court's decision to give movies First Amendment protection in 1952 put significant restrictions on film censorship. There were also changes within these censorship organizations in the mid-1950s that caused them to re-evaluate their approaches to censorship. Breen retired in 1954 and the PCA replaced him with Shurlock, a more liberal-minded regulator who questioned the contemporary relevance of the Production Code. During this same period Pope Pius XII questioned the Legion of Decency's focus on censoring movies and he called instead for a broader, more liberal, more positive approach to film by the Catholic Church. ${ }^{104}$ These changes led to a revision of the PCA's and the Legion's approaches that allowed some treatment (if tasteful) of previously banned topics like pregnancy, childbirth and abortion.

But from 1930 to 1958 the PCA and the Legion of Decency believed that these reproductive topics were ones that should not be dealt with in the entertainment media of cinema. The considered pregnancy a state of grace and a sacred obligation, but that this moral duty was restricted to married women only. Only children produced within the confines of heterosexual marriage represented an expression of God's love. As much as the censors cherished motherhood they strongly believed that pregnancy and childbirth were not appropriate subjects for motion pictures. For censors, pregnancy

100 Leff and Simmons, op. cit. (5), pp. 175-177.

101 Thomas F. Brady, 'Old order changes', New York Times, 23 July 1950, p. X5.

102 See Leff and Simmons, op. cit. (5), p. 173.

103 See Andrew Quicke, 'The era of censorship (1930-1967)', in John Lyden (ed.), The Routledge Companion to Religion and Film, New York: Routledge, 2009, pp. 32-51.

104 See Gregory D. Black, The Catholic Crusade against the Movies, 1940-1975, Cambridge: Cambridge University Press, 1998, pp. 175-181. 
was not only an intensely private affair, but also an unpleasant biological process whose entertainment value was questionable. This perception led the censors into categorizing films about pregnancy and childbirth as 'educational' and separating them from other types of 'entertainment' film. Pregnancy and childbirth were to be celebrated, but not seen.

Censorship organizations based their approach to movie censorship on simplistic assumptions about the nature of movies and the nature of communication. Rather than considering cinema as an artistic medium with complex signifiers of meaning, they believed that movies were essentially visual novels that produced meaning exclusively through narrative. From their perspective, films told linear stories using a heightened visual realism that conveyed easily understandable narratives to a monolithic audience. From this simplistic viewpoint cinema seemed to be a powerful medium of propaganda. By controlling the content of scripts the censors believed they could ensure that movies disseminated only morally appropriate messages, including those about pregnancy and childbirth. In the pre-Code era, censors had found narrative uncertainty to be a useful way of blunting 'indecent' messages about subjects like abortion. But after 1934 censorship groups tried to control the ambiguity in these cinematic stories so that audiences could only interpret a film's message in a single way.

Controversial aspects of post-conception reproduction put studios and censors into catch-22 situations. Censors' belief that cinematic depictions significantly influenced morality meant that they thought that certain stories about reproduction should never be told through this medium. They might approve of a story's anti-abortion message, but they felt that any cinematic portrayal would visually legitimate the subject. At various points in the history of movie censorship, however, censors were forced to perform narrative gymnastics using veiled language and ambiguous situations in order to try and tell some semblance of the story that studios wanted to tell about these forbidden subjects.

Although the administrators of the PCA and Legion of Decency were primarily men, there were also a significant number of women involved in their operation. These men and women were not trying to destroy the movie industry. Their goal was to reduce immorality and promote change for a healthier society by exerting their own cultural and moral influence. They strongly believed that they were acting in a parental role by protecting audiences from immoral and indecent films. But movie censorship essentially involved these religiously oriented elitist groups deciding for all women which aspects of female reproduction were suitable cinematic subjects. These censors' decisions were built on the assumption that women need to be protected from their own bodies. They believed that audiences would find authentic depictions of pregnancy and childbirth to be horrifying and indecent. They also worried that realistic portrayals of pregnancy and childbirth would scare young women away when it came time for them to perform their sacred duty of bearing children. These groups tried to make pregnancy and childbirth invisible by favouring fantasy versions of motherhood as opposed to real-world representations of conception, pregnancy and childbirth. Ultimately, movie censorship organizations believed that the reality of pregnancy and childbirth turned any film into a horror film. 